\title{
Statistics for management of complexity in electromagnetism
}

\author{
Joe Wiart • Luk Arnaut • Walid Tabbara
}

(C) Institut Télécom and Springer-Verlag 2011

Electromagnetic Fields (EMFs) have been studied and used for a long time. The past decades have seen a tremendous increase of applications of EMF in daily life. A particularly prevalent application is formed by wireless personal communication devices, e.g., mobile phones with currently more than five billion users worldwide. Large efforts have been expended to develop tools and methods that can be used to design EMF systems, to manage their performance, capabilities, quality, efficiency, compatibility, as well as to ensure that they comply with safety standards. Taking advantage of improvements in computer performance, efficient numerical methods continue to be developed that are being used to predict or characterize the EMF. In addition, particularly significant advances in software implementation and in experimental setups and techniques have been achieved during the past 15 years. Today, computers are capable of handling physically and electrically large problems, almost without limitations, thanks to distributed computing. Numerical methods such as FDTD

J. Wiart $(\bowtie)$

WHIST Lab, Orange Lab,

3840 rue du Général Leclerc,

92794 Issy les Moulineaux Cedex, France

e-mail: joe.wiart@orange-ftgroup.com

\section{Arnaut}

Department of Electrical and Electronic Engineering,

South Kensington Campus, Imperial College,

London SW7 2AZ, UK

e-mail: 1.arnaut@imperial.ac.uk

W. Tabbara

Laboratoire Signaux et Systèmes-DRE, Supélec,

3 rue Joliot-Curie,

91192 Gif-sur-Yvette Cedex, France

e-mail: tabbara@1ss.supelec.fr
(Finite-Difference Time-Domain) have been improved and adapted to allow for accurate estimation of EMFs in complex structures. On the experimental side, reverberation chambers have been studied and appear to be well suited to study various applications in electromagnetic compatibility and beyond.

Most, if not all, numerical and experimental methods and simulation tools have been designed mainly with a view to solve deterministic problems. In a mathematical framework, these can be defined as well-posed problems, characterized by differential equations for single-mode systems furnished with a complete set of fixed initial and boundary conditions. However, increasingly complex systems need to be handled and solved nowadays, in which one or more of the system parameters or boundary conditions may be ill-defined, i.e., be either unknown or fluctuating in a quasi-random manner, particularly for multi-mode dynamic systems. In addition to an estimate for the solution, a quantitative evaluation and statement of the associated uncertainty in the knowledge of the field quantity of interest is then called for. Computers that are capable of solving deterministic problems with millions of unknowns can provide only approximate solutions to a real physical process, but they cannot handle complex real-life scenarios involving uncertainty. On the other hand, in a physical experiment, the process output can be observed when the input is varying due to uncertainties. In other words, the uncertainties of the system's input parameters "drive" those in the output field variables.

Therefore, new approaches to numerical simulation are called for. Worldwide efforts are currently carried out in numerical as well as in experimental approaches in an effort to manage this situation through fusion of experimental, analytical, and statistical-physical methods of characterization. For example, geostatistics are being used to estimate 
the human exposure induced by radiating sources, to model uncertain electromagnetic environments by canonical stochastic fields, to use empirical mode decomposition of information fusion to extract, to compute and to interpret statistical moments in EM analysis, to name just a few.

Against this background, this special issue of Annals of Telecommunications provides the reader with a selection of invited articles on the latest developments in the field of managing complex problems in electromagnetism.

A paper by F. Mangeant presents an overview of joint initiatives regarding uncertainty management conducted in the frame of the ANR OPUS project, and the on-going activities in different working groups, giving some indications about the maturity of uncertainty management from different perspectives.

This is followed by a first group of papers dealing with the use of statistics in the assessment of human body exposure to electromagnetic fields. O. Aouiaz et al. address the analysis of the uncertainty of the specific absorption rate (SAR) induced in a phantom using a stochastic spectral collocation method. Kientega et al. describe a surrogate model to assess the whole-body SAR induced by multiple plane waves at $2.4 \mathrm{GHz}$. Jouvie et al. deal with the computation of the field radiated by a FM transmitter by means of ordinary kriging.

A second group of papers considers the use of statistics for field characterization. Amador et al. discuss a scheme for empirical statistical detection of non-ideal field distri- bution in a reverberation chamber and which is confirmed by a simple numerical model based on image theory. Orjubin et al. address the experimental determination of the maximum electric field level inside an overmoded reverberation chamber based on the generalized extreme value distribution. Gradoni et al. discuss the probability distribution and statistics of the minimum value of random electromagnetic fields for modeling deep fading in EM environments for wireless communications (dynamic multipath), including reverberation chambers for EMC.

Finally, a third set of papers deals with the use of statistical approaches in urban propagation and cablecoupling problems. Thomas et al. discuss the estimation of the probability distributions for cable-coupling using unscented transforms. Mostarshedi et al. present a statistical study on the influence of building architectural properties on scattered fields in an urban environment.

It is hoped that this special issue will stimulate further research in this burgeoning field. Management of complexity will always involve much more than bruteforce large-scale numerical simulations and data fusion. Ultimately, the link with the electromagnetics problem at hand requires a complete analysis, understanding and interpretation of data with inherent uncertainty and fluctuations that requires appropriate physical and engineering models. The work presented in this special issue is a step towards achieving this goal. We thank the authors for their valuable contribution. 